\title{
ENDOTRIVIAL MODULES FOR THE SYMMETRIC AND ALTERNATING GROUPS
}

\author{
JON F. CARLSON, NADIA MAZZA, AND DANIEL K. NAKANO
}

\begin{abstract}
In this paper we determine the group of endotrivial modules for certain symmetric and alternating groups in characteristic $p$. If $p=2$, then the group is generated by the class of $\Omega^{n}(k)$ except in a few low degrees. If $p>2$, then the group is only determined for degrees less than $p^{2}$. In these cases we show that there are several Young modules which are endotrivial.
\end{abstract}

\section{IntRoduction}

Endotrivial modules play an important role in the modular representation theory of finite groups. They are the building blocks of the endo-permutation modules and are an essential part of the Picard group of self equivalences of the stable category of $k G$-modules in the case that $G$ is a finite group and $k$ is a field of characteristic $p$. A few years ago, the first author and Jacques Thévenaz completed a program to classify the endotrivial $k G$-modules over $p$-groups [10]. Building on this result, Bouc [7] has completed a similar program to classify the endo-permutation modules over $p$-groups.

More recently, using the classification for $p$-groups, the authors of this paper started a project to classify the endotrivial modules over families of finite simple groups and related groups (cf. [8]). For a finite group of Lie type in the defining characteristic, we have found the structure of the group of endotrivial modules and explicit generators for the group in many cases. The group of endotrivial modules is cyclic, generated by the class of the syzygy module $\Omega(k)$, except in the cases that the group has Lie rank 1, or that the Lie rank is 2 and the field of definition of the group is small.

The natural next case, which we introduce with this paper, are the groups of endotrivial modules for the symmetric and alternating groups. In spite of the advanced nature of the representation theory of symmetric groups, determining the torsion in the endotrivial group is considerably more difficult than the case for groups of Lie type. To this point, we have only partial results. In particular, we give complete classifications with generators for the symmetric and the alternating groups, $\Sigma_{n}$ and $A_{n}$, on $n$ letters, in the cases that $p=2$, or that $p>2$ and $n<p^{2}$.

Date: November 22, 2007.

2000 Mathematics Subject Classification. 20C20, 20 C30.

Research of the first two authors was supported in part by NSF grant DMS-0401431.

Research of the third author was supported in part by NSF grant DMS-0400548. 
Let $k$ be a field of characteristic $p$ which is a splitting field for a given finite group $G$ and all its subgroups, and let $T(G)$ denote the group of endotrivial $k G$-modules. In this paper we prove the following.

Theorem A: Let $\Sigma_{n}$ be the symmetric group on $n$ letters.

(a) If $p=2$, then

$$
T\left(\Sigma_{n}\right) \cong \begin{cases}\{0\} & \text { if } n \leq 3, \\ \mathbb{Z}^{2} & \text { if } n=4,5 \\ \mathbb{Z} & \text { if } n \geq 6 .\end{cases}
$$

(b) If $p \geq 3$ and $1 \leq n<2 p$, then

$$
T\left(\Sigma_{n}\right) \cong \begin{cases}\{0\} & \text { if } n<p \\ \mathbb{Z} / 2(p-1) \mathbb{Z} & \text { if } n=p, p+1, \\ \mathbb{Z} / 2(p-1) \mathbb{Z} \oplus(\mathbb{Z} / 2 \mathbb{Z}) & \text { if } p+2 \leq n<2 p .\end{cases}
$$

(c) If $p \geq 3$ and $2 p \leq n<p^{2}$, then

$$
T\left(\Sigma_{n}\right) \cong \begin{cases}\mathbb{Z} \oplus(\mathbb{Z} / 2 \mathbb{Z})^{2} & \text { if } 2 p \leq n<3 p \\ \mathbb{Z} \oplus(\mathbb{Z} / 2 \mathbb{Z}) & \text { if } 3 p \leq n<p^{2}\end{cases}
$$

(d) If $p \geq 3$ and $p^{2} \leq n$, then

$$
\text { the torsion free rank of } T\left(\Sigma_{n}\right) \text { is } \begin{cases}2 & \text { if } p^{2} \leq n<p^{2}+p \\ 1 & \text { if } p^{2}+p \leq n\end{cases}
$$

Theorem B: Let $A_{n}$ be the alternating group on $n$ letters.

(a) If $p=2$, then

$$
T\left(A_{n}\right) \cong \begin{cases}\{0\} & \text { if } n \leq 3, \\ \mathbb{Z} \oplus(\mathbb{Z} / 3 \mathbb{Z}) & \text { if } n=4,5 \\ \mathbb{Z}^{2} & \text { if } n=6,7, \\ \mathbb{Z} & \text { if } n \geq 8 .\end{cases}
$$

(b) If $p \geq 3$ and $1 \leq n<2 p$, then

$$
T\left(A_{n}\right) \cong \begin{cases}\{0\} & \text { if } n<p, \\ \mathbb{Z} /(p-1) \mathbb{Z} \oplus(\mathbb{Z} / 2 \mathbb{Z}) & \text { if } n=p, p+1, \\ \mathbb{Z} / 2(p-1) \mathbb{Z} & \text { if } p+2 \leq n<2 p .\end{cases}
$$

(c) If $p \geq 3$ and $2 p \leq n<p^{2}$, then

$$
T\left(A_{n}\right) \cong \begin{cases}\mathbb{Z} \oplus(\mathbb{Z} / 4 \mathbb{Z}) & \text { if } p=3 \text { and } n=6,7, \\ \mathbb{Z} \oplus(\mathbb{Z} / 2 \mathbb{Z})^{2} & \text { if } p>3 \text { and } n=2 p, 2 p+1 \\ \mathbb{Z} \oplus(\mathbb{Z} / 2 \mathbb{Z}) & \text { if } 2 p+2 \leq n<3 p \\ \mathbb{Z} & \text { if } 3 p \leq n<p^{2}\end{cases}
$$

(d) If $p \geq 3$ and $p^{2} \leq n$, then

the torsion free rank of $T\left(A_{n}\right)$ is $\begin{cases}2 & \text { if } p^{2} \leq n<p^{2}+p \\ 1 & \text { if } p^{2}+p \leq n\end{cases}$ 
In the case that $p=2$, the problem is reasonably straightforward because the Sylow 2-subgroups are self normalizing except in a couple of well known cases. These results are found in Section 4. A surprise in the odd characteristic case for the symmetric groups is that there are several Young modules which are endotrivial.

We strongly suspect that for $p>2$ and $n \geq p^{2}$, the groups $T\left(\Sigma_{n}\right)$ and $T\left(A_{n}\right)$ have no torsion, beyond that coming from the sign representation. The torsion free ranks of these groups are known from general principals that give us the results stated above. That is, the torsion free rank of $T(G)$ depends only on the number of $G$ conjugacy classes of maximal elementary abelian $p$-subgroups of order $p^{2}$. However, we do not know (yet) how to determine generators for the torsion free part of $T(G)$ unless this rank is 1 , or, if $p=2$ and the Sylow 2-subgroups are dihedral. For our purposes, this concerns the groups $T\left(\Sigma_{n}\right)$ and $T\left(A_{n}\right)$ for $p \geq 3$ and $p^{2} \leq n<p^{2}+p$. In addition, finding the torsion elements of $T\left(\Sigma_{n}\right)$ and $T\left(A_{n}\right)$ for $p \geq 3$ and $p^{2} \leq n$ have not been completed as of this writing. We plan to investigate these issues in future work.

Several of our calculations for small groups relied on the algebra software MAGMA (cf. [6]). The computations turned out to be very effective in revealing to us that there are nontrivial endotrivial modules whose class is a torsion element in the group of endotrivial modules. This led us to understand and prove the underlying theoretical facts.

We are grateful to David Hemmer for providing us with several useful insights about symmetric group representations during an Oberwolfach meeting in March 2006 .

\section{Setting}

Throughout the paper, let $G$ be a finite group, usually the symmetric group $\Sigma_{n}$ or the alternating group $A_{n}$ acting on the set $\{1, \ldots, n\}$, for some $n$, and let $k$ be a field of characteristic $p$ which is a splitting field for $G$ and all of its subgroups. For this, it is sufficient that $k$ contains all $m^{\text {th }}$ roots of unity where $m$ is the order of $G$. When defining subgroups of the symmetric or alternating groups we assume the natural ordering on the letters unless otherwise indicated. For example, we write $H=$ $\Sigma_{a} \times \Sigma_{b} \subseteq \Sigma_{n}$ to mean the subgroup where $\Sigma_{a}$ is the collection of all permutations on $\{1, \ldots, a\}$ and $\Sigma_{b}$ is the set of all permutations in $\Sigma_{n}$ on $\{a+1, \ldots, a+b\}$ $(a+b \leq n)$.

For two subgroups $H$ and $K$ of $G$ let $[G / H]$ denote a complete set of representatives for the left $H$-cosets in $G$ and let $[H \backslash G / K]$ be a complete set of representatives for the $H-K$ double cosets in $G$. Normally, we assume that the identity element 1 of $G$ is one of the representatives, so that $[G / H] \backslash\{1\}$ means a set of representatives of the nonidentity cosets.

We consider only finitely generated left modules over group algebras. If $M$ is a $k G$-module, we write $\operatorname{Res}_{H}^{G} M$, or $M \downarrow_{H}^{G}$, for the restriction of $M$ to $k H$ where $H$ is a subgroup of G. If $N$ is another $k G$-module, we denote by $\operatorname{Hom}_{k}(M, N)$ the $k G$ module of all $k$-linear maps $M \longrightarrow N$. We let $\operatorname{End}_{k} M=\operatorname{Hom}_{k}(M, M)$ denote the $k$-endomorphism ring of $M$. The $k$-dual of $M$ is $M^{*}=\operatorname{Hom}_{k}(M, k)$, where $k$ also denotes the trivial $k G$-module. Let $M \otimes N$ be the tensor product of two modules $M$ 
and $N$ over the base field $k$ with diagonal action of the group $G$. We write $M \mid N$ to mean that the module $M$ is a direct summand of $N$.

For $M$ a $k G$-module, we denote the kernel of the projective cover $P \longrightarrow M$ by $\Omega(M)$ and the cokernel of the injective hull $M \rightarrow Q$ by $\Omega^{-1}(M)$. Inductively, we define for any $n>1, \Omega^{n}(M)=\Omega\left(\Omega^{n-1}(M)\right)$ and $\Omega^{-n}(M)=\Omega^{-1}\left(\Omega^{1-n}(M)\right)$. The combinatorics are that $\left(\Omega^{n}(M)\right)^{*} \cong \Omega^{-n}\left(M^{*}\right)$ and that, for any module $N$, there exists a projective module $P$ such that $\Omega^{n}(M) \otimes N \cong \Omega^{n}(M \otimes N) \oplus P$.

We write $\bmod (k G)$ for the category of all finitely generated $k G$-modules and $\operatorname{stmod}(k G)$ for the stable module category, namely the quotient of $\bmod (k G)$ by the subcategory of projective modules. That is, the stable category has the same objects as $\bmod (k G)$. For two finitely generated $k G$-modules $M$ and $N$, the morphisms from $M$ to $N$ in the stable category are given by

$$
\underline{\operatorname{Hom}}_{k G}(M, N)=\operatorname{Hom}_{k G}(M, N) / \operatorname{PHom}_{k G}(M, N),
$$

where $\operatorname{PHom}_{k G}(M, N)$ is the subspace of homomorphisms that factor through a projective module.

Definition 2.1. A $k G$-module $M$ is endotrivial provided $\operatorname{End}_{k} M \cong k \oplus$ (proj), or equivalently, $\operatorname{End}_{k} M \cong k$ in $\operatorname{stmod}(k G)$.

Recall that $\operatorname{Hom}_{k}(M, N) \cong M^{*} \otimes N$ as $k G$-modules. Consequently, the tensor product of two endotrivial modules is endotrivial. This allows us to define the group of endotrivial modules, whose elements are classes $[M]$ as follows.

Definition 2.2. Two endotrivial $k G$-modules are equivalent if they are isomorphic in $\operatorname{stmod}(k G)$. That is, $[M]=[N]$ if $M \oplus P \cong N \oplus Q$ for projective modules $P$ and $Q$. The group of endotrivial $k G$-modules is the set $T(G)$ of equivalence classes $[M]$ of endotrivial $k G$-modules $M$, with the operation given by the rule $[M]+[N]=[M \otimes N]$.

Clearly, $T(G)$ is abelian, and we have that $0=[k]$ and $-[M]=\left[M^{*}\right]$. Furthermore, if $p$ does not divide the order of $G$, then every module is projective. In this case, the definition of an endotrivial module does not have much meaning, as every object in the stable category is equivalent to the zero object, and also every module is an endotrivial module, by a strict interpretation of the definition. In that case, we set $T(G)=\{0\}$.

The next theorem collects some useful properties of the group $T(G)$.

Theorem 2.3. Let $G$ be any finite group. The group $T(G)$ is finitely generated. Thus, there is a torsion-free subgroup $T F(G)$ of finite rank, such that $T(G) \cong$ $T T(G) \oplus T F(G)$, where $T T(G)$ denotes the torsion subgroup of $T(G)$.

(a) The modules $\Omega^{n}(k)$ are endotrivial and their classes generate a cyclic direct summand of $T(G)[1,11,12,10]$.

(b) Let $n$ denote the number of conjugacy classes of maximal elementary abelian p-subgroups of p-rank 2 in $G$. Then the rank of $T F(G)$ is $n$ if $G$ has p-rank at most 2 and is $n+1$ if the p-rank of $G$ is greater than $2[1,8]$.

(c) If $E$ is an abelian p-group having p-rank at least 2 , then $T(E) \cong \mathbb{Z}$ and is generated by the class of $\Omega(k)[11,12]$. 
(i) If $G$ has p-rank at least 2 , then the product of the restriction maps

$$
\text { Res : } T F(G) \longrightarrow \prod_{E} T(E)
$$

from $T F(G)$ to all of the elementary abelian p-subgroups $E$ of p-rank 2 of $G$, is injective $[1,8]$.

(ii) A $k G$-module is endotrivial if and only if its restriction to every elementary abelian p-subgroup of $G$ is endotrivial [10].

(d) Let $P$ be a Sylow p-subgroup of $G$.

(i) The torsion subgroup $T T(P)$ is trivial except in the case that $P$ is cyclic, quaternion or semidihedral [10].

(ii) If $T T(P)$ is trivial, then $T T(G)$ is generated by the classes $[M]$ of indecomposable endotrivial $k G$-modules $M$ such that $M \downarrow_{P}^{G} \cong k \oplus$ (proj), for a projective $k P$-module (proj) [8]. (Note that in general, a module with vertex $P$ and trivial source is not endotrivial.)

(e) The restriction map $\operatorname{Res}_{H}^{G}: T(G) \longrightarrow T(H)$ is injective, provided the subgroup $H$ of $G$ contains the normalizer of a Sylow p-subgroup of $G$ [8].

It follows from (d) in the above theorem that the torsion subgroup $T T(P)$ of $T(P)$ is often trivial. We will show in the next sections that, as a consequence of this, then $T T(G)$ is generated by the equivalence classes of the indecomposable trivial source modules that are endotrivial, for an arbitrary finite group $G$ having a Sylow $p$-subgroup isomorphic to $P$. If $G$ is the symmetric group, it turns out that some of these modules can be found among the Young modules. We refer the reader to [18] for the basic background on the representation theory of the symmetric groups, and to [14], [16] and [17] for deeper investigations of the Young modules. Some computer computations of Young module can be found on the first author's web page (http://www.math.uga.edu/ ${ }^{\sim}$ jfc/hecke.html).

Definition 2.4. Let $\lambda=\left(\lambda_{1}, \ldots, \lambda_{k}\right)$ be the a partition of $n$ and let $G=\Sigma_{n}$.

(a) A Young subgroup $\Sigma_{\lambda}$ associated to $\lambda$ is a subgroup of $G$ that is conjugate to $\Sigma_{\lambda_{1}} \times \cdots \times \Sigma_{\lambda_{k}}$.

(b) The Specht module $S^{\lambda}$ associated to $\lambda$ is the $k G$-module with $k$-basis the set of standards polytabloids (cf. [18]). It is a submodule of the permutation module $M^{\lambda}=k \uparrow_{\Sigma_{\lambda}}^{G}$.

(c) The Young module $Y^{\lambda}$ associated to $\lambda$ is the unique (up to isomorphism) indecomposable direct summand of $M^{\lambda}$ that contains $S^{\lambda}$.

It should also be mentioned that all of the indecomposable direct summands of the permutation modules $M^{\lambda}$ are Young modules $Y^{\mu}$ for some partitions $\mu$ which are greater than or equal to $\lambda$ in the dominance ordering. In particular, we will call upon the following observation.

Lemma 2.5. Suppose that $S$ is a Young subgroup of $G=\Sigma_{n}$ that contains a Sylow p-subgroup of $G$. Suppose that $N$ is an indecomposable $k G$-module such that $N \downarrow_{S}^{G} \cong$ $k \oplus$ (proj). Then $N$ is a Young module with vertex a Sylow p-subgroup of $G$. 
Proof. Because $S$ contains a Sylow $p$-subgroup, $N$ is relatively $S$-projective. Consequently, $N$ is a direct summand of $N \downarrow_{S}^{G} \uparrow_{S}^{G}$. Since $k$ is a direct summand of $N \downarrow_{S}^{G}$, then a vertex of $N$ is a Sylow $p$-subgroup of $G$, and $N$ is a direct summand of $k \uparrow_{S}^{G}$.

Remark 2.6. Note that in the proof of the above lemma, $N$ is a Young module simply because every indecomposable summand of $k \uparrow \uparrow_{S}^{G}$ is a Young module. We can not conclude that $N$ is the Young module corresponding to the same partition as the Young subgroup $S$. Indeed, the trivial module $N=k$ satisifies the hypotheses of the Lemma, even when the subgroup $S$ corresponds to a non-trivial partition.

\section{Some Generalities}

In this section we present a few general results that will be used in the course of this paper.

Proposition 3.1. Suppose that $H$ is a normal subgroup of $G$ and that $p$ does not divide the index of $H$ in $G$. Let $M$ be an indecomposable endotrivial $k G$-module. Then $M \downarrow_{H}^{G}$ is endotrivial and indecomposable.

Proof. Assume that $H$ is normal in $G$ and that $M$ is an endotrivial indecomposable $k G$-module. Then $M \downarrow_{H}^{G} \cong M_{0} \oplus Q$, with $M_{0}$ an indecomposable endotrivial module and $Q$ a projective module. Since $p$ does not divide $|G: H|, M$ is projective relative to $H$ and we deduce that $M$ is a direct summand of $M_{0} \uparrow_{H}^{G}$. It follows that $M \downarrow_{H}^{G}$ is a direct summand of $M_{0} \uparrow_{H}^{G} \downarrow_{H}^{G} \cong \bigoplus_{g \in[G / H]}{ }^{g} M_{0}$, since $H \triangleleft G$. The only way that this can happen is if $Q$ is the zero module.

Lemma 3.2. Let $H$ be a subgroup of $G$ that contains a Sylow p-subgroup $P$ of $G$. If $|P|>|G: H|$, then the kernel of the restriction map $\operatorname{Res}_{H}^{G}: T(G) \longrightarrow T(H)$ is generated by the classes of the one dimensional $k G$-modules $M$ such that $M \downarrow_{H}^{G}=k$. In particular, if $G=\Sigma_{n}, H=\Sigma_{n-1}, n>2 p$ and if $p \nmid n$, then the restriction map $\operatorname{Res}_{H}^{G}: T(G) \longrightarrow T(H)$ is injective. The same applies if $G=A_{n}$ and $H=A_{n-1}$. Similarly, if $p>2$, then the kernel of the restriction map $T\left(\Sigma_{n}\right) \longrightarrow T\left(A_{n}\right)$ has order 2 and is generated by the class of the sign representation.

Proof. Let $M$ be an indecomposable endotrivial $k G$-module such that $M_{H}^{G} \cong k \oplus$ (proj). Then, by relative projectivity and a vertex argument, we have that $M \mid k \uparrow_{H}^{G}$. Since $\operatorname{Dim}\left(k \uparrow_{H}^{G}\right)=|G: H|<|P|$, the $k H$-module $M \downarrow_{H}^{G}$ has no nonzero projective summand. Therefore, $\operatorname{Dim}(M)=1$.

Assume that $G=\Sigma_{n}$ and $H=\Sigma_{n-1}$ (resp. $G=A_{n}$ and $H=A_{n-1}$ ), with $n>2 p$. Assume also that $p \nmid n$. Then, the index $|G: H|=n<|P|$, where $P$ is a common Sylow $p$-subgroup of $G$ and $H$. Hence, $\operatorname{Ker}\left(\operatorname{Res}_{H}^{G}\right)$ consists of the isomorphism classes (in $\operatorname{stmod}(k H)$ ) of the 1-dimensional $k G$-modules that restrict trivially to $H$. If $G=\Sigma_{n}$ or $G=A_{n}$, then there is exactly one such module: the trivial module. Likewise, $\operatorname{Ker}\left(\operatorname{Res}_{A_{n}}^{\Sigma_{n}}\right)$ is generated by the class of the sign representation if $p>2$. 
We will now provide some general information about the situation when the field has characteristic 2. The following proposition is recorded in [3, Lemma 5.4, Theorem 5.5].

Proposition 3.3. Suppose that $G$ is a finite group whose Sylow 2-subgroups are dihedral. Then $G$ has two conjugacy classes of elementary abelian 2-subgroups of rank 2 , represented by subgroups $E_{1}$ and $E_{2}$. Let $P_{0}$ be the projective cover of the trivial $k G$-module $k$. Then taking the radical modulo the socle of $P_{0}$ (i.e. the "heart" of $P_{0}$ ) we get

$$
\operatorname{Rad}\left(P_{0}\right) / \operatorname{Soc}\left(P_{0}\right) \cong M \oplus M^{*}
$$

where $M \downarrow_{E_{1}}^{G} \cong \Omega(k)$ and $M \downarrow_{E_{2}}^{G} \cong \Omega^{-1}(k)$. Hence, $M$ is an endotrivial module and

$$
T F(G)=\langle[\Omega(k)],[M]\rangle \cong \mathbb{Z}^{2} .
$$

Moreover, if a dihedral group $D$ is a Sylow 2-subgroup of $G$, then the restriction map $\operatorname{Res}_{H}^{G}: T F(G) \longrightarrow T(D)$ is an isomorphism.

Proposition 3.3 provides the answer to a question that was left open in [8]. Namely, in the case that $G=\mathrm{PSL}_{3}\left(\mathbb{F}_{2}\right)$ (i.e. the finite group of Lie type $A_{2}(2)$ ), we have that the Sylow 2-subgroups are dihedral of order 8. In [8, Theorem 8.1], we demonstrated that $T(G) \cong \mathbb{Z}^{2}$. Now, the diagram of the projective cover $P_{0}$ of the trivial module is (cf. [5, Section 11])

$$
M_{k}{ }^{k} M^{*}, \quad \text { with } M \text { of dimension } 3 .
$$

So, we have $\operatorname{Rad}\left(P_{0}\right) / \operatorname{Soc}\left(P_{0}\right) \cong M \oplus M^{*}$. By Proposition 3.3, $M$ is endotrivial and we have $T(G)=\langle[\Omega(k)],[M]\rangle$.

We end this section with a known result about the normalizers of the Sylow 2subgroups of the symmetric and alternating groups.

Proposition 3.4. Let $n$ be a positive integer.

(a) The Sylow 2-subgroups of $\Sigma_{n}$ are self normalizing for all $n$.

(b) The Sylow 2-subgroups of $A_{n}$ are self normalizing for all $n \geq 6$.

Proof. This is a result due to Louis Weisner, cf. [21, Corollary 2, Theorem p.124].

\section{THE CASE $p=2$}

In this section, we give a complete characterization of the group $T(G)$ in the case that $G=\Sigma_{n}$ and $G=A_{n}$ for $p=2$. For each family of groups, we first determine the isomorphism type of $T(G)$, and then describe the generators in detail.

Theorem 4.1. Suppose that $G=\Sigma_{n}$ and $p=2$. Then $T T(G)=\{0\}$, for all positive integers $n$. We have that

$$
T(G) \cong T F(G) \cong \begin{cases}\{0\} & \text { if } n \leq 3, \\ \mathbb{Z}^{2} & \text { if } n=4,5 \\ \mathbb{Z} & \text { if } n \geq 6 .\end{cases}
$$


Proof. Let $P$ be a Sylow $p$-subgroup of $G$. In this case, $N:=N_{G}(P)=P$, by Proposition 3.4. By the classification of endotrivial modules of a $p$-group (cf. [10]), we know that $T T(P)=\{0\}$. Indeed, $P$ is either cyclic of order 2 , dihedral of order 8 , or has all maximal elementary abelian 2-subgroups of 2-rank at least 3 , in the three cases. The last statement is an exercise for the reader. If $n=6$ or 7 , then $P$ is a direct product of a cyclic group of order 2 with a dihedral group of order 8 , and the conclusion about the ranks of the maximal elementary abelian subgroups is obvious. If $n=8$ or 9 , then $P$ is a wreath product of a dihedral group of order 8 and a cyclic group of order 2, and again the conclusion holds. For larger $n$, we always have that $P$ is a direct product of wreath products of Sylow subgroups of smaller symmetric groups.

Because $N=P$, we have that $T T(N)=\{0\}$, and so $T T(G)=\{0\}$. The result for $T(G)$ is then a direct consequence of Theorem 2.3 and of [8, Corollary 3.2].

Let us now explore the group $T(G)$ further. First, note that in the theorem, if $T(G)$ is cyclic, then $T(G)=\langle[\Omega(k)]\rangle$, and this tells us all about $T(G)$, for $n \geq 6$ or $n \leq 3$. When $n=4$ or 5 , the class $[\Omega(k)]$ generates one summand of $T(G)$, and, because $P$ is dihedral, there are at least two ways of finding a generator for the other summand. For one we know the structure of the projective cover $P_{0}$ of the trivial module $k$, as in Proposition 3.3. The structure of these modules can be seen from the diagrams in [5]. The other method is to draw on the classical representation theory of the symmetric group, which turns out to be a matter of linear algebra.

Say $P=\left\langle x, y \mid x^{2}=y^{2}=(x y)^{4}=1\right\rangle$, so that the center of $P$ is generated by the element $(x y)^{2}$. By $\left[9\right.$, Theorem 5.4], we have that $T(P)=\left\langle[\Omega(k)],\left[\Omega_{P /\langle x\rangle}(k)\right]\right\rangle \cong \mathbb{Z}^{2}$, where $\Omega_{P /\langle x\rangle}(k)$ is the kernel of the map $k[P /\langle x\rangle] \longrightarrow k$ sending each coset $u\langle x\rangle$ to 1 , and $k[P /\langle x\rangle]$ is the permutation $k P$-module with $k$-basis the cosets of $P /\langle x\rangle$, on which $P$ acts by left multiplication.

On the other hand, the Specht module $S^{(3,1)}$ has dimension 3 and we can take as $k$-basis the set of $(3,1)$-polytabloids, i.e. the row equivalence classes of the standard $(3,1)$-tableaux. We refer the reader to [18] for the details.

A direct computation shows that the $k P$-modules $S^{(3,1)} \downarrow_{P}^{G}$ and $\Omega_{P /\langle x\rangle}(k)$ are isomorphic, and hence it proves that

$$
T(G)=\left\langle[\Omega(k)],\left[S^{(3,1)}\right]\right\rangle .
$$

Let us now consider $G=\Sigma_{5}$ and let $G^{\prime}=\Sigma_{4}<G$. Then, by [20, Lemma 1.5], we have that $S^{(3,1)} \uparrow_{G^{\prime}}^{G} \cong S^{(4,1)} \oplus M$, where $M$ is an extension

$$
0 \longrightarrow S^{(3,1,1)} \longrightarrow M \longrightarrow S^{(3,2)} \longrightarrow 0 \text {. }
$$

Thus, $M$ has dimension 11, and is the Green correspondent of $S^{(3,1)}$ over $k G$. It is also the Green correspondent of $\Omega_{P /\langle x\rangle}(k)$. Thus, by $[3, \S 6]$, we have that $M$ is endotrivial and $M \downarrow_{P}^{G} \cong \Omega_{P /\langle x\rangle}(k) \oplus k P$. This shows that

$$
T(G)=\langle[\Omega(k)],[M]\rangle,
$$

where $M$ is the above module. 
For the alternating groups we have the following. Again, the case of $n \leq 3$ is trivial, since then 2 does not divide the order of $A_{n}$.

Theorem 4.2. Suppose that $G=A_{n}$, the alternating group on $n$ letters, and $p=2$. The group of endotrivial modules has the form

$$
T(G) \cong \begin{cases}\{0\} & \text { if } n \leq 3 \\ \mathbb{Z} \oplus(\mathbb{Z} / 3 \mathbb{Z}) & \text { if } n=4,5, \\ \mathbb{Z}^{2} & \text { if } n=6,7, \\ \mathbb{Z} & \text { if } n \geq 8 .\end{cases}
$$

Proof. Let $P$ be a Sylow 2-subgroup of $G$. If $n=4$ or 5 , then $P$ is isomorphic to a Klein four group and the normalizer $N$ of $P$ has the form $P \rtimes C_{3}$, where $C_{3}$ is a cyclic group of order 3 . Hence $T F(G)=T F(N) \cong \mathbb{Z}$ and so

$$
T(N)=T F(N) \oplus T T(N) \cong \mathbb{Z} \oplus(\mathbb{Z} / 3 \mathbb{Z}) .
$$

Indeed, since $k$ is a splitting field for $N$, the group $T T(N)$ identifies with the character group of $N$, which is isomorphic to $\mathbb{Z} / 3 \mathbb{Z}$. Now, in both cases, $P$ is a TI (trivial intersection) subgroup of $G$. That is, if $x \in G$ and $x \notin N_{G}(P)$, then $x P x^{-1} \cap P=\{1\}$. In such a situation, the stable module categories $\operatorname{stmod}(k G)$ and $\operatorname{stmod}(k N)$ are equivalent, by the induction and restriction functors, and these functors induce isomorphisms $T(G) \cong T(N)$ (cf. [8, Proposition 2.8]). Hence we have that $T(G) \cong \mathbb{Z} \oplus(\mathbb{Z} / 3 \mathbb{Z})$.

For $n>5$, the Sylow 2-subgroups $P$ of $G$ are self normalizing (cf. Proposition 3.4). Consequently, there are no nontrivial one dimensional representations of the normalizer $N=P$ of $P$. It follows that $T T(G)=T T(N)=\{0\}$. In the cases that $n=6$ or 7 , the Sylow 2-subgroups are dihedral of order 8 and hence we have that $T(G)=T F(G) \cong \mathbb{Z}^{2}$, by Theorem 2.3. In all other cases, $G$ has no maximal elementary abelian 2-subgroup of rank 2 .

As for the symmetric group, let us look for the additional generator of $T F\left(A_{n}\right)$ needed when $n=6,7$. For this, we use Proposition 3.3, since $P$ is dihedral, and we consider the diagrams of the projective cover of the trivial module given in [4, Appendix pp. 206 and 213]. It turns out that there are endotrivial modules of dimension 19 if $n=6$, and of dimension 35 if $n=7$ that are the $k A_{n}$-Green correspondents of the $k P$-module $\Omega_{P /\langle x\rangle}(k)$, as defined above. In particular, we observe that the restriction map $\operatorname{Res}_{P}^{G}: T(G) \longrightarrow T(P)$ is an isomorphism.

\section{THE CASE $n<2 p$, FOR $p \geq 3$}

From this point on we assume that $p \geq 3$ (i.e. $p$ is an odd prime). Notice first that if $n<p$ then $p$ does not divide the order of $G=\Sigma_{n}$, nor of $A_{n}$, and every module is projective. Thus, $T(G)=\{0\}$ if $n<p$, as mentioned in Section 2. We now consider the case when $p \leq n<2 p$.

Proposition 5.1. Let $G=\Sigma_{n}$, and let $N=N_{G}(P)$, where $P$ is a Sylow p-subgroup of $G$. 
(a) If $n=p$ or $n=p+1$, then

$$
T(G)=T T(G) \cong T T(N) \cong \mathbb{Z} / 2(p-1) \mathbb{Z} .
$$

(b) If $n=p+b$, with $1<b<p$, then

$$
T(G)=T T(G) \cong T T(N) \cong \mathbb{Z} / 2(p-1) \mathbb{Z} \oplus(\mathbb{Z} / 2 \mathbb{Z}) .
$$

Proof. Suppose that $p \leq n<2 p$. Then $P$ is cyclic of order $p$, and so $T(P)=$ $T T(P)=\langle[\Omega(k)]\rangle \cong \mathbb{Z} / 2 \mathbb{Z}$. The subgroup $P$ is a TI subgroup of $G$. Hence the restriction functor induces an isomorphism $T(G) \cong T(N)$ (cf. [8, Proposition 2.8]).

In the case that $n=p$ or $n=p+1$, we have that $N \cong C_{p} \rtimes C_{p-1}$, and $T T(N) \cong$ $\mathbb{Z} / 2(p-1) \mathbb{Z}$, since we assume that $k$ is a splitting field for $N$. That is, by a routine calculation it can be shown that $\Omega^{2}(k)$ is a one dimensional $k N$-module which is a generator for the multiplicative group of one dimensional $k N$-modules (one dimensional characters of $N$ ). Consequently, the class of $\Omega(k)$ generates a cyclic subgroup of order $2(p-1)$ in $T(N)$. But this must be all of $T(N)$ since we know that the kernel of the restriction map from $T(N)$ to $T(P)$ is the group generated by the one dimensional $k N$-modules and that $T(P)$ has order 2 .

When $n=p+b$, for an integer $1<b<p$, we have that $N \cong\left(C_{p} \rtimes C_{p-1}\right) \times \Sigma_{b}$, and so $T T(N) \cong \mathbb{Z} / 2(p-1) \mathbb{Z} \oplus(\mathbb{Z} / 2 \mathbb{Z})$, where the $\mathbb{Z} / 2 \mathbb{Z}$ factor is the subgroup generated by the class of the inflation of the sign representation of $\Sigma_{b}$ to $N$.

Similar arguments apply to the alternating groups, and they lead us to the following result.

Proposition 5.2. Let $A=A_{n}$, and let $N_{A}=N_{A}(P)$, where $P$ is a Sylow p-subgroup of $A$.

(a) If $n=p$ or $n=p+1$, then

$$
T(A)=T T(A) \cong T T\left(N_{A}\right) \cong \mathbb{Z} /(p-1) \mathbb{Z} .
$$

(b) If $n=p+b$, with $1<b<p$, then

$$
T(A)=T T(A) \cong T T\left(N_{A}\right) \cong \mathbb{Z} / 2(p-1) \mathbb{Z} .
$$

Proof. We have, as above, $T(P)=T T(P)=\langle[\Omega(k)]\rangle \cong \mathbb{Z} / 2 \mathbb{Z}$. Because $P$ is a $\mathrm{TI}$ subgroup of $A$, we get that $T(A) \cong T\left(N_{A}\right)$. Now, embed $A$ into $G=\Sigma_{p+b}$ and consider $N=N_{G}(P)$ as in the previous proposition. By Lemma 3.2, the restriction map $T(N) \longrightarrow T\left(N_{A}\right)$ has kernel equal to $\mathbb{Z} / 2 \mathbb{Z}$. That is, the class of the sign representation is the only nontrivial element of the kernel. In the case that $b=0,1$, we get immediately that $T\left(N_{A}\right) \cong \mathbb{Z} /(p-1) \mathbb{Z}$ as asserted. Now suppose that $1<b<p$. Then, $N \cong\left(C_{p} \rtimes C_{p-1}\right) \times \Sigma_{b}$. Let $J=A_{n} \cap \Sigma_{b}$ where here, the $\Sigma_{b}$ means the $\Sigma_{b}$ factor in $N$ as we have expressed it. Note first that $J$ is in the kernel of every one dimensional representation of $N_{A}$ since it is in the commutator subgroup of $\Sigma_{b}$. Hence, $T\left(N_{A} / J\right) \cong T\left(N_{A}\right)$ by the inflation map. But now $N_{A} / J \cong C_{p} \rtimes C_{p-1}$. This proves the last statement. 


\section{The CASE $n=a p$, FOR AN INTEGER $2 \leq a<p$}

We begin this section with the description of the $p$-local group structure of the groups $G=\Sigma_{n}$ and $A=A_{n}$, in the case that $2 p \leq n<p^{2}$. Write $n=a p+b$, with $2 \leq a<p$ and $0 \leq b<p$. Note that $p$ must be an odd prime. We assume that $G$ acts on the set $\{1, \ldots, n\}$. Let $P$ be a common Sylow $p$-subgroup of $G$ and of $A$ having the form

$$
P=\langle(1, \ldots, p),(p+1, \ldots, 2 p), \ldots,((a-1) p+1, \ldots, a p)\rangle
$$

and let $N=N_{G}(P)$ be the normalizer of $P$ in $G$ and $N_{A}=N_{A}(P)=N \cap A$. For simplicity, if $X \subseteq G$, we denote by $X_{A}$ the intersection $X \cap A$.

Let us describe certain subgroups of $G$. We leave it to the reader to determine the structure of the corresponding subgroups for the alternating groups. The group $P$ is elementary abelian of $p$-rank $a$, and $N \cong N_{p} \imath \Sigma_{a} \times \Sigma_{b}$, where $N_{p} \cong C_{p} \rtimes C_{p-1}$ is the normalizer in $\Sigma_{p}$ of a Sylow $p$-subgroup of $\Sigma_{p}$. Here, "?" denotes the wreath product, and for a positive integer $\ell$, we denote by $C_{\ell}$ a (multiplicative) cyclic group of order $\ell$. We will also use the notation $X^{a}$ for the direct product of $a$ copies of a group $X$. Let $S$ denote the Young subgroup containing $P$ and corresponding to the partition $(p, \ldots, p, b)$ of $n$. Then $H=N S$ is the normalizer of $S$ in $G$ and it is isomorphic to $\Sigma_{p} \prec \Sigma_{a} \times \Sigma_{b}$.

Hence, we have the following (3-dimensional) diagram of the inclusions of these subgroups (with obvious identifications of the subgroups of $G$ ):

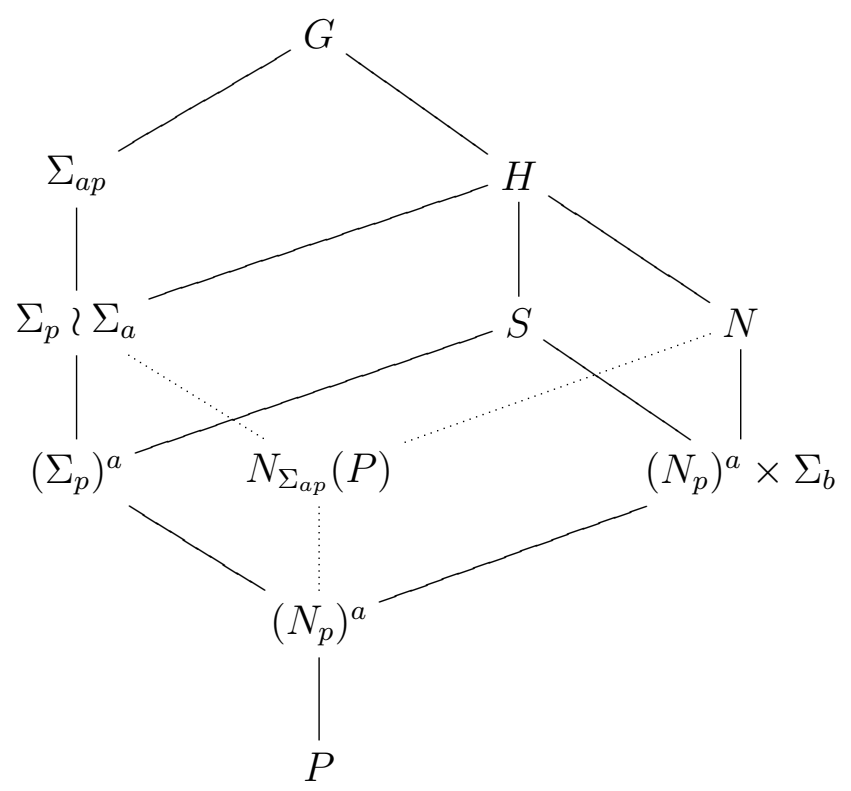

In this section, we consider the case in which $n=a p$, for an integer $2 \leq a<p$. For convenience, we will denote by $N_{S}=N_{S}(P) \cong\left(N_{p}\right)^{a}$.

Because $P$ is elementary abelian of $p$-rank at least 2, TT(P) is trivial. Thus, $T T(N)$ and $T T\left(N_{A}\right)$ are generated by the isomorphism classes of 1-dimensional $k N$ - and $k N_{A}$-modules, respectively, by Theorem 2.3. Since the arguments for $A$ and for $G$ are alike, we will only work through the case of the symmetric group 
and state the corresponding results for the alternating groups, without rewriting the reasoning.

From $N \cong N_{p} 2 \Sigma_{a}$, we deduce that $T T(N) \cong \mathbb{Z} /(p-1) \mathbb{Z} \oplus(\mathbb{Z} / 2 \mathbb{Z})$, where the $\mathbb{Z} /(p-1) \mathbb{Z}$ and $\mathbb{Z} / 2 \mathbb{Z}$ factors correspond to the subgroups generated by the $k$-linear characters of $N_{p}$ and by the sign representation of $\Sigma_{a}$ respectively.

Let $X$ be a $k N$-module of dimension one, and let $Y$ be the $k H$-Green correspondent of $X$. This makes sense because $N \subseteq H$. Then let $X_{N_{S}}=X \downarrow_{N_{S}}^{N}$, and since $N_{S}=N_{S}(P)$, it makes sense to consider the $k S$-Green correspondent $Y_{S}$ of $X_{N_{S}}$. Matching the modules with the corresponding groups, we get the following pictures (where the double headed dotted arrows stand for the Green correspondence between the indicated modules and groups)
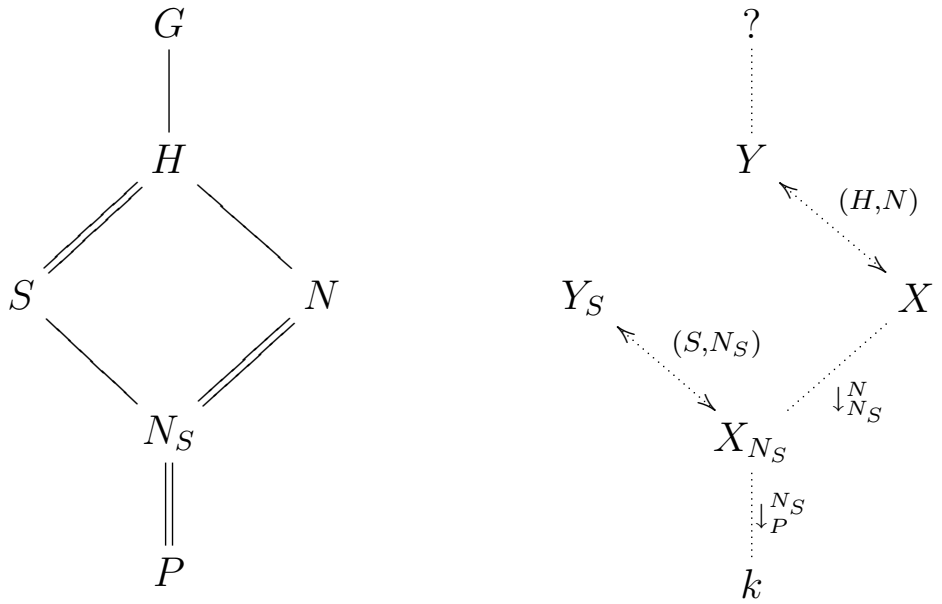

Our aim now is to limit the choices for the module $Y_{S}$.

Lemma 6.1. If $Y$ is an endotrivial $k H$-module, then $Y_{S}$ is an endotrivial $k S$-module and $Y \downarrow_{S}^{H} \cong Y_{S}$.

Proof. By a property of the Green correspondence $Y$ is a direct summand of the induced module $X \uparrow_{N}^{H}$. Thus, using the Mackey formula and the equality $H=N S$, we get that $Y \downarrow_{S}^{H}$ is a direct summand of

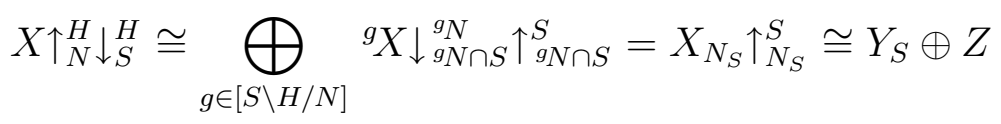

where $Z$ is a direct sum of indecomposable $k S$-modules each having vertex strictly contained in $P$. We remind the reader that $[S \backslash H / N]$ is a complete set of representative of the $S$ - $N$ double cosets in $H$. Since $Y$ is indecomposable with vertex $P$, its restriction $Y \downarrow_{S}^{H}$ to $S$ must have $Y_{S}$ as direct summand. Consequently, if we assume that $Y$ is endotrivial, then $Y_{S}$ must also be endotrivial and the other direct summands appearing in the decomposition of $Y \downarrow_{S}^{H}$ are projective. That is, because $Y$ is endotrivial, its restriction to $S$ is endotrivial and can have only one non-projective indecomposable component. Now, the facts that $S \triangleleft H$ and $p \nmid|H: S|$ imply, by Proposition 3.1, that $Y \downarrow_{S}^{H} \cong Y_{S}$. 
By the contrapositive of the lemma, if $Y_{S}$ is not endotrivial, then neither is $Y$. So we are down to the question of finding criteria for $Y_{S}$ to be endotrivial. This is the main result of the section.

Proposition 6.2. The module $Y_{S}$ is endotrivial if and only if $\operatorname{Dim}\left(Y_{S}\right)=1$.

Proof. The "if" part is clear. So now assume that $Y_{S}$ is endotrivial. Let $K=$ $N_{p} \times \Sigma_{p}{ }^{a-1} \subseteq G$, where the $i^{\text {th }}$-factor $\Sigma_{p}$ acts on $\{i p+1, \ldots,(i+1) p\}$, for $1 \leq i \leq a-1$ and where $N_{p}$ is the normalizer of $\langle(1 \ldots p)\rangle$ in the subgroup $\Sigma_{p}$ of $S$ that acts on $\{1, \ldots, p\}$. Thus, we have $N_{S}<K<S$. Because $Y_{S}$ is an endotrivial module, we may write $Y_{S} \downarrow_{K}^{S} \cong U \oplus V$ for $k K$-modules $U$ and $V$ with $U$ indecomposable endotrivial and $V$ projective. Moreover, the Green correspondence implies that $U \downarrow_{N_{S}}^{K} \cong X_{N_{S}} \oplus W$ for some projective $k N_{S^{-}}$module $W$ (since $U$ is endotrivial). We also have that $U$ is a direct summand of $X_{N_{S}} \uparrow \stackrel{K}{N_{S}}$. Moreover,

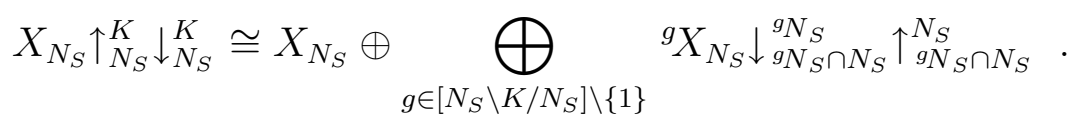

Since the largest normal $p$-subgroup $O_{p}(K)$ of $K$ is nontrivial and is contained in $N_{S}$, none of the indecomposable factors of the right hand side is projective. From the fact that $U$ is endotrivial, we deduce that $U \downarrow_{N_{S}}^{K} \cong X_{N_{S}}$. Therefore, $\operatorname{Dim}(U)=1$.

Now, $Y_{S}$ is a direct summand of $U \uparrow T_{K}^{S}$, by the Green correspondence, which applies since $N_{S} \subseteq K$. Therefore, $Y_{S} \downarrow_{K}^{S}$ is a direct summand of

$$
U \uparrow_{K}^{S} \downarrow_{K}^{S} \cong U \oplus \bigoplus_{g \in[K \backslash S / K] \backslash\{1\}}{ }^{g} U \downarrow_{g_{K} \cap K}^{g_{K}} \uparrow_{g_{K K} \cap K}^{K}
$$

Again, $g$ runs over a set of representatives of the $K$ - $K$-double cosets in $S$, such that $g \notin K$. Now, notice that we may take for $K$-coset representatives $[S / K]$ in $S$ the $N_{p}$-cosets representatives $\left[\Sigma_{p} / N_{p}\right]$ in $\Sigma_{p}$ of the corresponding normal subgroups of $S$ and $K$ respectively. Thus, we may assume that every coset representative centralizes the subgroup $\left(\Sigma_{p}\right)^{a-1}$ of $K$. It follows that every intersection ${ }^{g} K \cap K$, for $g \in[K \backslash S / K]$ contains a nontrivial $p$-subgroup. Hence, the right hand side in the displayed isomorphism does not contain any projective summands. Since $Y_{S}$ is endotrivial, we deduce that $Y_{S} \downarrow_{K}^{S} \cong U$. Hence $\operatorname{Dim}\left(Y_{S}\right)=1$ as claimed.

The last two results have an immediate consequence.

Corollary 6.3. Let $M$ be an indecomposable endotrivial $k G$-module (where $G=\Sigma_{a p}$ $2 \leq a<p$ ) whose class belongs to the torsion subgroup TT $(G)$. Then, $M_{\downarrow_{H}}^{G} \cong$ $Y \oplus$ (proj), where $Y$ is an endotrivial $k H$-module of dimension 1.

By very similar arguments for the alternating group $A=A_{a p}$, with $2 \leq a<p$, we prove the following.

Corollary 6.4. Let $M$ be an indecomposable endotrivial $k A$-module whose class belongs to the torsion subgroup $T T(A)$. Then, $M \downarrow_{H_{A}}^{A} \cong Y \oplus(\operatorname{proj})$, where $Y$ is an endotrivial $k H_{A}$-module of dimension 1 . 


\section{THE CASE $n=2 p$}

In this section, we apply the results of Section 6 in the case that $G=\Sigma_{2 p}$ and $A=A_{2 p}$. As noted, $T(P)=\langle[\Omega(k)]\rangle \cong \mathbb{Z}$. We assume the notation of Section 6 for $G$ and for $A$, and since the reasonings for $A$ and for $G$ are alike, we only give the details for $G$ and leave the alternating case as exercise for the reader.

By $[2,6.1-2]$ the subgroup $H$ is strongly $p$-embedded in $G$, that is ${ }^{g} H \cap H$ is a $p^{\prime}$-group for any $g \in G \backslash H$. Therefore, for any $k H$-module $Y$ of dimension 1 we have that

$$
Y \uparrow_{H}^{G} \downarrow_{P}^{G} \cong Y \downarrow_{P}^{H} \oplus \bigoplus_{g \in[P \backslash G / H] \backslash\{1\}}{ }^{g} Y \downarrow_{g_{H} \cap P}^{g_{H}} \uparrow^{{ }^{P}}{ }_{g_{H} \cap P}=k \oplus \bigoplus_{g \in[P \backslash G / H] \backslash\{1\}} k^{\uparrow_{g_{H}}^{P} \cap P}=k \oplus \text { (proj) }
$$

where the sums are over representatives of the nonidentity $P$ - $H$-double cosets. The isomorphism holds because for $g \notin H$ the intersection ${ }^{g} H \cap P$ is trivial. Moreover, the one dimensional modules for $H \cong \Sigma_{p} 2 \Sigma_{2}$ form a Klein four group. We may take for generators the $k H$-modules $\varepsilon_{H}$ and $Y=k_{\Sigma_{p}} \zeta \varepsilon_{\Sigma_{2}}$, where $\varepsilon_{K}$ denotes the sign representation of the group $K$.

Observe that the isomorphism $Y \downarrow_{S}^{H} \cong k$ forces the $k G$-Green correspondent $M$ of $Y$ to be the Young module $Y^{(p, p)}$, associated to the partition $(p, p)$ of $2 p$. Indeed, $M$ is an indecomposable nontrivial direct summand with vertex $P$ of the permutation module $k \uparrow_{S}^{G}$ (cf. [18] and [14]).

Proposition 7.1. Let $G=\Sigma_{2 p}$ for $p$ an odd prime. Then any one dimensional $k H$-module induced to $G$ is endotrivial. The group $T(G)$ is given by

$$
T(G)=\left\langle[\Omega(k)],\left[\varepsilon_{G}\right],\left[Y^{(p, p)}\right]\right\rangle \cong \mathbb{Z} \oplus(\mathbb{Z} / 2 \mathbb{Z})^{2} .
$$

Using this result we get the following.

Proposition 7.2. Let $A=A_{2 p}$ for $p$ an odd prime. Then any one dimensional $\mathrm{kH}_{A}$-module, when induced to $A$, is endotrivial. The group $T(A)$ is given by

$$
T(A)=\left\langle[\Omega(k)],\left[Y^{(p, p)} \downarrow_{A}^{G}\right],[M]\right\rangle \cong \begin{cases}\mathbb{Z} \oplus(\mathbb{Z} / 2 \mathbb{Z})^{2} & \text { if } p>3 \\ \mathbb{Z} \oplus(\mathbb{Z} / 4 \mathbb{Z}) & \text { if } p=3\end{cases}
$$

where $M$ is an indecomposable endotrivial $k A$-module such that $M \uparrow_{A}^{G}$ is indecomposable.

The last claim above comes from the nature of the 1-dimensional $k H_{A}$-modules. Namely, if $E \cong A_{p} \times A_{p}$ then $E \subseteq H$ and is also a subgroup of $H_{A}$. It is easy to see that the restriction of any one dimensional $k H_{A}$-module $Y$ to $E$ is trivial. Thus, $Y$ is a $k\left[H_{A} / E\right]$-module. Since the quotient $H / E$ is a dihedral group of order 8 and $H_{A}$ has index 2 in $H$, we have either that $Y$ extends to $H$, or that the induction $Y \uparrow_{H_{A}}^{H}$ is indecomposable. In the first case, this means that $Y$ is the restriction of a $k H$-module, and so we get $Y^{(p, p)} \downarrow_{A}^{G}$ as $k A$-Green correspondent. Otherwise, $Y_{H_{A}}^{H}$ is indecomposable, which implies that the $k A$-Green correspondent $M$ of $Y$ has the property that $M \uparrow{ }_{A}^{G}$ is indecomposable. We refer the reader to Section 8 for further details on the structure of $M$. 
Note that, if $p=3$, then $H_{A} / E$ is cyclic of order 4 , and so $T T(A) \cong T T\left(H_{A}\right) \cong$ $\mathbb{Z} / 4 \mathbb{Z}$, since we assume that $k$ is a splitting field for $H_{A}$. In particular, this shows that $Y^{(p, p)} \downarrow_{A}^{G} \cong M \otimes M$ in $\operatorname{stmod}(k A)$.

Before leaving this case we record the following fact for later application.

Proposition 7.3. For the group $H=\Sigma_{p} 2 \Sigma_{2}$, we have that

$$
T T(H)=\left\langle\left[\varepsilon_{H}\right],[Y]\right\rangle \cong(\mathbb{Z} / 2 \mathbb{Z})^{2}
$$

where $\varepsilon_{H}$ is the sign representation on $H$ and $Y$ is the one dimensional module on which $\Sigma_{p} \times \Sigma_{p}$ acts trivially and $\Sigma_{2}$ acts by the sign representation.

Proof. It is clear that $\varepsilon_{H}$ and $Y$ are endotrivial modules, and moreover, they generate the group of one dimensional $k H$-modules. The only question is whether there are other endotrivial modules generating $T T(H)$. So, suppose that $U$ is an indecomposable endotrivial $k H$-module whose restriction to $P$ is isomorphic to $k \oplus$ (proj). By Lemma 6.1 and Proposition 6.2, the $k S$-module $U \downarrow_{S}^{H}$ is an indecomposable endotrivial module and has dimension 1 . Hence the class $[U]$ is in the subgroup of $T(G)$ genereated by $\varepsilon_{H}$ and $[Y]$.

\section{CASE $n=2 p+b$ FOR $0<b<p$}

In this section, we consider the cases $G=\Sigma_{2 p+b}$ and $A=A_{2 p+b}$, for an integer $b$ with $0<b<p$. Throughout this section we use the notation for $G$ and for $A$ given at the beginning of Section 6 .

Again, $T F(G)=\langle[\Omega(k)]\rangle$ and $T F(A)=\langle[\Omega(k)]\rangle$ are infinite cyclic, because the Sylow $p$-subgroup $P$ is elementary abelian of order $p^{2}$. By Theorem 2.3, since $T(P)$ is torsion-free, the groups $T T(G)$ and $T T(A)$ are generated by the classes of indecomposable modules whose restriction to $N=N_{G}(P)$ and $N_{A}$ respectively, are isomorphic to the direct sum of a one dimensional module and a projective module. In the case of the symmetric group, we show that these modules are Young modules, or the sign representation. Then, we will easily deduce a set of generators for $T(G)$. The situation for the alternating groups is more complicated and hence it is handled separately. Before splitting the question into subcases, we point out the following fact which shows that $T T(G)$ and $T T(A)$ have at most order 4 .

Lemma 8.1. In the above notation, the restriction maps $\operatorname{Res}_{\Sigma_{2 p}}^{G}: T(G) \longrightarrow T\left(\Sigma_{2 p}\right)$ and $\operatorname{Res}_{A_{2 p}}^{A}: T(A) \longrightarrow T\left(A_{2 p}\right)$ are injective, for all $1 \leq b \leq p-1$.

Proof. The proof is immediate, by the transitivity of the restriction map and by Lemma 3.2.

At this point, we handle separately the groups $G$ and $A$, and for simplicity, we start with $G$. Namely, we exhibit a nontrivial endotrivial module whose class is in $T T(G)$, and this will prove that $T T(G)$ is a Klein four group.

For convenience, let us set $G_{b}=\Sigma_{2 p+b}, H_{b}$ for the subgroup of the form $\left(\Sigma_{p} 2 \Sigma_{2}\right) \times$ $\Sigma_{b}$, and $S_{b} \cong \Sigma_{p}^{2} \times \Sigma_{b}$, for $0 \leq b<p$ (cf. Section 6$)$. 
Let $H$ and $Y$ be as in Proposition 7.3. We identify $H$ with the quotient $H_{b} / \Sigma_{b}$, and let $\widetilde{Y}$ and $\widetilde{\varepsilon}_{H}$ be the inflations of $Y$ and $\varepsilon_{H}$ to $H_{b}$, respectively. Since $\widetilde{Y} \downarrow_{S_{b}}^{H_{b}}=k$, the $k G_{b}$-Green correspondent $V$ of $\widetilde{Y}$ is isomorphic to a direct summand of the permutation module $M^{(p, p, b)}=k \uparrow_{S_{b}}^{G_{b}}$ (cf. Lemma 2.5). Hence $V$ is a Young module of the form $Y^{\lambda}$ for some partition $\lambda$ with $\lambda \unrhd(p, p, b)$. By [14, Theorem 2], the Young module $Y^{\lambda}$ associated to a partition $\lambda$ of $n$ is projective if and only if $\lambda$ is $p$-restricted. A partition $\lambda=\left(\lambda_{1}, \ldots, \lambda_{s}\right)$ of a positive integer $n$ is $p$-restricted (or $p$-column regular) if $\lambda_{i}-\lambda_{i+1}<p$, for all $1 \leq i<s$, and if $\lambda_{s}<p$. This restricts the number of possible endotrivial modules among all the Young modules.

Proposition 8.2. Let $G_{b}=\Sigma_{2 p+b}$ for an integer $b$ such that $0<b<p$. Consider the same notation as above and as in the Proposition 7.3. Let $\mu$ be the partition $(p+b, p)$ of $n$. Then we have the following.

(a) The Young module $Y^{\mu}$ is endotrivial.

(b) The $k G$-Green correspondent of $\widetilde{\varepsilon_{H}}$ is not endotrivial, and so

$$
T(G)=\left\langle[\Omega(k)],\left[\varepsilon_{G}\right],\left[Y^{\mu}\right],\right\rangle \cong \mathbb{Z} \oplus(\mathbb{Z} / 2 \mathbb{Z})^{2}, \forall 0 \leq b<p
$$

Proof. Let us first observe that (b) follows immediately from (a), as noted above in Lemma 8.1, and by Proposition 7.1. Hence, the only thing we need to show is that $Y^{\mu}$ is endotrivial.

We proceed by induction on $b$. We know that the result holds for $b=0$, by Proposition 7.1. Assume $b \geq 1$. We apply Theorem 5.1 in [17]. In the notation of Henke's article (where $k$ is an index, and not the underlying field), we have $r=2 p+b, k=p$ and $t(k)=0$, for all $0<b<p$. Thus, again in Henke's notation, $V_{p}=0$, implying that

$$
\operatorname{Res}_{G_{b-1}}^{G_{b}} Y^{\mu} \cong Y^{(p+b-1, p)} \oplus\left\{\begin{array}{ll}
Y^{(p+b, p-1)} & , \text { if } 0<b<p-1 \\
0 & , \text { if } b=p-1
\end{array} .\right.
$$

Now, the partition $(p+b, p-1)$ of $2 p+b-1$ is $p$-restricted for all integers $b$ such that $0<b<p-1$. In these cases, the $k G_{b-1}$-module $Y^{(p+b, p-1)}$ is projective (cf. [14, Theorem 2]), and $Y^{(p+b-1, p)}$ is endotrivial by the induction hypothesis and by Proposition 7.1. If $b=p-1$, then the $k G_{b-1}$-module $Y^{(2 p-2, p)}$ is not projective, since the partition $(2 p-2, p)$ is not $p$-restricted. However, it is isomorphic to the Specht module $S^{(2 p-2, p)}$, by the computation of $[13,(2.6)]$. Then, applying the modular branching rules (cf. [20]), the restricted $k G_{b-2}$-module $\operatorname{Res}_{G_{b-2}}^{G_{b-1}} S^{(2 p-2, p)}$ is still indecomposable, and hence, it is isomorphic to the Young module $Y^{(2 p-3, p)}$, which is endotrivial by the induction hypothesis. This shows that the Young $k G_{b^{-}}$ module we started with, namely $Y^{(2 p-1, p)}$ is endotrivial. This completes the case for the symmetric groups.

We now turn to the alternating groups. Let us first consider the group $A=A_{2 p+1}$. Write $G=\Sigma_{2 p+1}, G_{0}=\Sigma_{2 p}, A_{0}=A_{2 p}, H=\Sigma_{p} 2 \Sigma_{2}, H_{A}=H \cap A, S=$ 
$\Sigma_{p} \times \Sigma_{p}, S_{A}=S \cap A$ and $V=A_{p} \times A_{p}$. So we have the diagram:

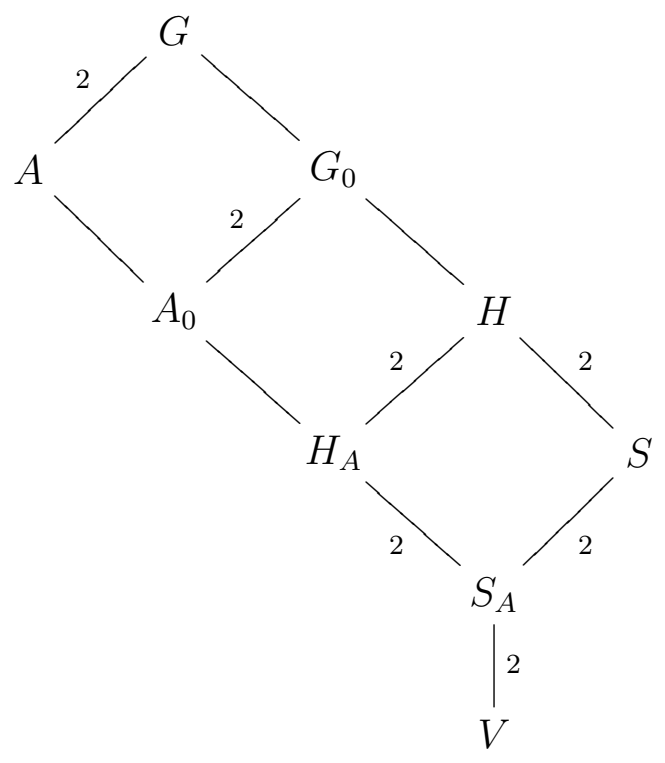

where a number on an edge indicates the index of the subgroup. Since the quotient $H / V$ is a dihedral group of order 8 , there is a simple $k H$-module $U$ of dimension 2 , whose restrictions to $H_{A}$ and $S$ split as a direct sum of two nonisomorphic modules. That is, a direct summand $U_{A}$ of $U \downarrow_{H_{A}}^{H}$ is a 1-dimensional module whose $k A$-Green correspondent might be endotrivial. We prove that this is indeed the case.

Observe that, by Proposition 7.2, the $k A_{0}$-Green correspondent of $U_{A}$ is endotrivial, and hence, the $k G_{0}$-Green correspondent of $U$ restricts to $A_{0}$ as a direct sum of two nonisomorphic endotrivial modules. On the other hand, a direct summand $U_{S}$ of $U \downarrow_{S}^{H}$ is isomorphic to the outer tensor product $k_{\Sigma_{p}} \otimes \varepsilon_{\Sigma_{p}}$ as $k \Sigma_{p} \otimes k \Sigma_{p^{-}}$ module (recall that $S=\Sigma_{p} \times \Sigma_{p}$ ). Consequently, the induced module $U_{S} \uparrow_{S}^{G}$ has a direct summand which is the signed Young module $Y^{(p+1 \mid p)}$ in the notation of [15]. Roughly, the signed Young $k \Sigma_{n}$-module $Y^{(a \mid b)}$ is defined as a certain uniquely determined indecomposable direct summand of the signed permutation module $M^{(a \mid b)}=\left(1_{\Sigma_{a}} \otimes \varepsilon_{\Sigma_{b}}\right) \uparrow_{\Sigma_{a} \times \Sigma_{b}}$, for a 2-parts partition $(a, b)$ of $n$. Now, by $\left[15\right.$, Proposition 3.6.1], we have that $Y^{(p+1 \mid p)} \cong S^{\left(p+1,1^{p}\right)}$, for the (ordinary) Specht module. By property of the Green correspondence (or by $[19,7.6]$ ), we have that $S^{\left(p+1,1^{p}\right)} \downarrow_{A}^{G} \cong M \oplus^{c} M$, for two nonisomorphic $k A$-modules $M$ and ${ }^{c} M$, and where we may assume that $M$ is the $k A$-Green correspondent of $U_{A}$. Upon restriction to $G_{0}$ and by the modular branching rules (cf. [20, Lemma 1.5]), we have that $S^{\left(p+1,1^{p}\right)} \downarrow_{G_{0}}^{G}$ is an indecomposable module isomorphic to a nonsplit extension $X$ given by the exact sequence

$$
0 \longrightarrow S^{\left(p, 1^{p}\right)} \longrightarrow X \longrightarrow S^{\left(p+1,1^{p-1}\right)} \longrightarrow 0
$$

and $X$ is isomorphic to the $k G_{0}$-Green correspondent of $U$. Also, $X$ is isomorphic to the signed Young module $Y^{(p \mid p)}$, and $X \downarrow_{A_{0}}^{G_{0}} \cong M_{0} \oplus^{c} M_{0}$, with $M_{0}$ and ${ }^{c} M_{0}$ endotrivial and nonisomorphic. Relating to the above picture of inclusion of subgroups of $G$, we can summarize the situation into a diagram (where the double headed dotted arrows 
stand for the Green correspondence between the indicated modules and groups)

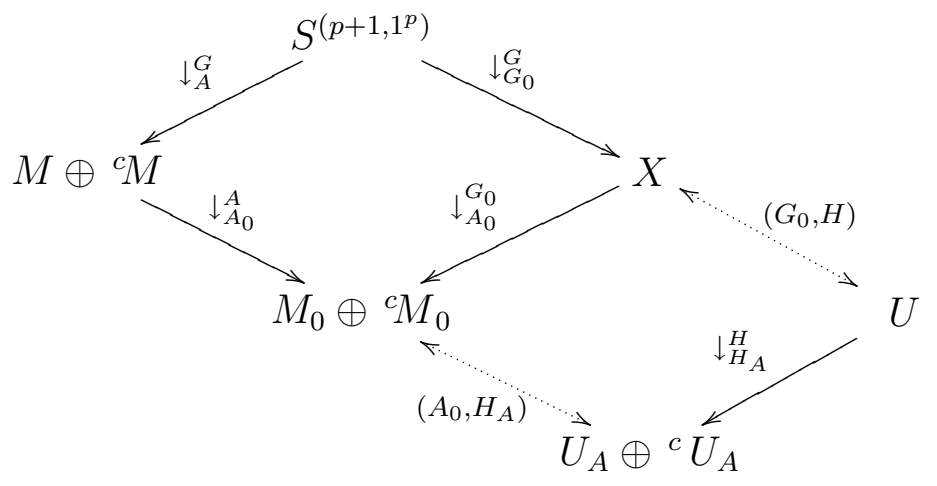

In particular, since $S^{\left(p+1,1^{p}\right)} \downarrow_{G_{0}}^{G}$ is indecomposable, the restriction $M \downarrow_{A_{0}}^{A}$ is also indecomposable, and we may assume (without loss of generality) that $M \downarrow_{A_{0}}^{A} \cong M_{0}$. The fact that $M_{0}$ is endotrivial implies that $M$ is also endotrivial (since $M$ extends $M_{0}$ to $A$ ). This proves the following.

Proposition 8.3. Let $A=A_{2 p+1}$ and $G=\Sigma_{2 p+1}$. Then,

$$
T(A)=\left\langle[\Omega(k)],\left[Y^{(p+1, p)} \downarrow_{A}^{G}\right],[M]\right\rangle \cong \mathbb{Z} \oplus(\mathbb{Z} / 2 \mathbb{Z})^{2},
$$

where $M$ is an indecomposable direct summand of the restriction to $A$ of the Specht $k G$-module $S^{\left(p+1,1^{p}\right)}$. Moreover, $M \downarrow_{A_{2 p}}^{A}$ is indecomposable.

Let us now consider the group $A=A_{2 p+2}$. Then $A$ contains a group $G^{\prime}$ isomorphic to $\Sigma_{2 p}$, and $A$ is contained in $G=\Sigma_{2 p+2}$. We show the following.

Lemma 8.4. The restriction map $\operatorname{Res}_{G^{\prime}}^{A}: T(A) \longrightarrow T\left(G^{\prime}\right)$ is injective and the cokernel has order 2 , generated by the class of the sign representation $\varepsilon_{G^{\prime}}$ of $G^{\prime}$.

Proof. Let $M$ be an indecomposable endotrivial $k A$-module such that $M \downarrow_{G^{\prime}}^{A} \cong k \oplus$ (proj). By relative projectivity and a vertex argument, we deduce that $M$ is a direct summand of $k \uparrow_{G^{\prime}}^{A}$. Thus, $M$ is a direct summand of

$$
k \uparrow_{G^{\prime}}^{G} \downarrow_{A}^{G} \cong M^{(2 p, 1,1)} \downarrow_{A}^{G}, \quad \text { since } G^{\prime} \cong \Sigma_{2 p} \subseteq G .
$$

Now, $M^{(2 p, 1,1)} \cong \oplus_{\mu} Y^{\mu}$ for some partitions $\mu$ of $2 p+2$ such that $\mu \unrhd(2 p, 1,1)$. Thus, $M$ is a direct summand of $Y^{\mu}$ for some such $\mu$. Since $M$ has vertex a Sylow $p$ subgroup and is a trivial source module, it must be that $Y^{\mu}$ has complexity 2. By [16, Theorem 3.3.2], this leaves us with only two candidates, namely $Y^{(2 p+2)}=k$, whose Green correspondent is the trivial module, and $Y^{(2 p+1,1)} \cong S^{(2 p+1,1)}$ (the Specht module corresponding to the natural permutation representation of $G$ ). In the second case, it is known that the restriction of $S^{(2 p+1,1)}$ to $A$ is indecomposable (cf. $[19,7.6]$ ) and has dimension $2 p+1$ (cf. [18]). Therefore, the latter has no endotrivial summand. This proves the injectivity of the restriction map $\operatorname{Res}_{G^{\prime}}^{A}$.

The statement for the cokernel can be proved by using a argument similar to the one used to verify the injectivity of the restriction. Indeed, let $M$ be an indecomposable endotrivial $k A$-module such that $M \downarrow_{G^{\prime}}^{A} \cong \varepsilon_{G^{\prime}} \oplus$ (proj). Then, we notice 
that $M$ does not extend to $G$. Otherwise, we would have that $M=N \downarrow_{A}^{G}$ for $N$ an indecomposable endotrivial $k G$-module, which would be one of the modules $\varepsilon_{G}$, $Y^{(p+2, p)}$ or $\varepsilon_{G} \otimes Y^{(p+2, p)}$. But $\varepsilon_{G} \downarrow_{G^{\prime}}^{G} \cong \varepsilon_{G} \downarrow_{A}^{G} \downarrow_{G^{\prime}}^{A}=k$ and $Y^{(p+2, p)} \downarrow_{G^{\prime}}^{G} \cong Y^{(p, p)} \oplus$ (proj), by transitivity of the restriction. Thus, $M \uparrow_{A}^{G} \downarrow_{A}^{G} \cong M \oplus{ }^{c} M$, with $M \nsubseteq{ }^{c} M$. Thus, ${ }^{c} M \downarrow_{G^{\prime}}^{A} \cong k \oplus$ (proj) and so, by the above argument, we conclude that if such an $M$ exists, then $M$ has dimension 1 . Hence, $M$ does not exist, as was to be shown.

Now Lemmas 3.2 and 8.4 imply the following.

Proposition 8.5. Let $A=A_{2 p+b}$ and $G=\Sigma_{2 p+b}$, for an integer $b$ with $1<b<p$. Then $T(A)$ is the image of the restriction $\operatorname{map} \operatorname{Res}_{A}^{G}: T(G) \longrightarrow T(A)$, that is

$$
T(A)=\left\langle[\Omega(k)],\left[Y^{(p+b, p)} \downarrow_{A}^{G}\right]\right\rangle \cong \mathbb{Z} \oplus(\mathbb{Z} / 2 \mathbb{Z}) .
$$

9. CASE $n=a p+b$ FOR $3 \leq a<p$ AND $0 \leq b<p$

In this section we determine $T(G)$ and $T(A)$ for all groups $G=\Sigma_{a p+b}$ and $A=$ $A_{a p+b}$, such that $3 \leq a<p$ and $0 \leq b<p$. As in most of the work, the two can be handled alike, and in order to avoid repetitions, we state the results for both groups and we only make explicit the reasoning for the symmetric groups, leaving the translation to the alternating group as an exercise for the reader.

Again we have that $T(P)=\langle[\Omega(k)]\rangle \cong \mathbb{Z}$, since $P$ is an elementary abelian group of $p$-rank $a$. We begin with the case that $b=0$.

Proposition 9.1. Let $G=\Sigma_{a p}$ and $A=A_{a p}$, for an integer a such that $3 \leq a<p$. Then

$$
T(G)=\left\langle[\Omega(k)],\left[\varepsilon_{G}\right]\right\rangle \cong \mathbb{Z} \oplus(\mathbb{Z} / 2 \mathbb{Z}) \quad \text { and } \quad T(A)=\langle[\Omega(k)]\rangle \cong \mathbb{Z} .
$$

Proof. By Proposition 7.3, we have that $T T(H)=\left\langle\left[\varepsilon_{H}\right],[Y]\right\rangle \cong(\mathbb{Z} / 2 \mathbb{Z})^{2}$, where $Y=$ $k_{\Sigma_{p}} \prec \varepsilon_{\Sigma_{a}}$. We also know from Theorem 2.3 that the map $\operatorname{Res}_{H}^{G}: T T(G) \longrightarrow T T(H)$ is injective. Note that the $k G$-Green correspondent of $\varepsilon_{H}$ is $\varepsilon_{G}$, which is endotrivial. Let $M$ be the $k G$-Green correspondent of $Y$. The only question is whether $M$ is endotrivial. Note also that $Y \downarrow_{S}^{H} \cong k$ implies that $M$ is a Young module that is a nontrivial direct summand with vertex $P$ of the permutation module $M^{\lambda}=k \uparrow_{S}^{G}$, where $\lambda=(p, \ldots, p)$.

Let $U=\Sigma_{p} \times \Sigma_{(a-1) p}$ be the subgroup of $G$ containing $S$, with $\Sigma_{p}$ acting on $\{1, \ldots, p\}$ and $\Sigma_{(a-1) p}$ on $\{p+1, \ldots, a p\}$. We set $K=U \cap H \cong \Sigma_{p} \times\left(\Sigma_{p}\left\langle\Sigma_{(a-1)}\right)\right.$ and $L=K \cap N$. Hence, $L=N_{U}(P)$ and $S \subseteq K$.

Assume that $M$ is endotrivial. Then, $M \downarrow_{U}^{\bar{G}}=M_{U} \oplus$ (proj) for an indecomposable endotrivial $k U$-module $M_{U}$, and $M \downarrow_{K}^{G} \cong Y_{K} \oplus$ (proj), where $Y_{K}=Y \downarrow_{K}^{H}$. By transitivity of the restriction, we deduce then that $M_{U} \downarrow_{K}^{U} \cong Y_{K} \oplus$ (proj). More precisely, $Y_{K}$ and $M_{U}$ are Green correspondents, since $K \supseteq L=N_{U}(P)$. In particular, $M_{U}$ is a direct summand of $Y_{K} \uparrow_{K}^{U}$, and by the Mackey formula,

$$
Y_{K} \uparrow_{K}^{U} \downarrow_{K}^{U} \cong Y_{K} \oplus \bigoplus_{g \in[K \backslash U / K] \backslash\{1\}}{ }^{g} Y_{K} \downarrow^{g_{K}}{ }_{g_{K} \cap K} \uparrow_{g_{K} \cap K}^{K}
$$


By choice of the subgroups $U$ and $K$, we have that $p$ divides $\left|{ }^{g} K \cap K\right|$, for any $g \in U$, and so the right hand side has no projective summand. Therefore, $M_{U} \downarrow_{K}^{U} \cong Y_{K}$. In particular, $M_{U}$ has dimension one.

In addition, since $p$ does not divide $|G: U|$, the $k G$-module $M$ is a nonprojective module that is relatively projective to $U$. Hence, $M \downarrow_{U}^{G}$ is a direct summand of

$$
M_{U} \uparrow_{U}^{G} \downarrow_{U}^{G} \cong M_{U} \oplus \bigoplus_{g \in[U \backslash G / U] \backslash\{1\}}{ }^{g} M_{U} \downarrow_{g_{U}}^{g_{U}}{ }^{G} \uparrow_{g U \cap U}^{U} .
$$

Note that $p$ divides $\left|{ }^{g} U \cap U\right|$, for all $g \in G$, since the intersection ${ }^{g} U \cap U$ contains a subgroup $\Sigma_{(a-2) p}$ for all $g \in G$. Therefore, the right-hand side has no projective summand. Hence, the assumption $M \downarrow_{U}^{G}=M_{U} \oplus$ (proj) implies that $M \downarrow_{U}^{G}=M_{U}$, and so $M$ has dimension one. Since the only one dimensional $k G$-modules are isomorphic to $k$ or $\varepsilon_{G}$, we have proved the claim for $G$.

Now, for $A$, we observe that the same arguments as for $G$ work. This implies that $T T(A)$ is trivial, since there is no nontrivial 1-dimensional $k A$-module.

We now apply Lemma 8.1 in order to determine the endotrivial modules in the remaining cases.

Corollary 9.2. Let $G=\Sigma_{a p+b}$ and $A=A_{a p+b}$ for $3 \leq a<p$ and $0 \leq b<p$. Then

$$
T(G)=\left\langle[\Omega(k)],\left[\varepsilon_{G}\right]\right\rangle \cong \mathbb{Z} \oplus(\mathbb{Z} / 2 \mathbb{Z}) \quad \text { and } \quad T(A)=\langle[\Omega(k)]\rangle \cong \mathbb{Z} .
$$

\section{REFERENCES}

[1] J. Alperin, A construction of endo-permutation modules, J. Group Theory 4 (2001), 3-10.

[2] M. Aschbacher, Simple connectivity of p-group complexes, Isr. J. Math. 82 (1993), 1-43.

[3] M. Auslander and J. Carlson, Almost split sequences and group algebras, J. Algebra, 103 (1986), 122-140.

[4] D. Benson, Modular Representation Theory: New Trends and Methods, Lecture Notes in Mathematics No. 1081, Springer, Berlin, 1984.

[5] D. Benson and J. Carlson, Diagrammatic methods for group representations and cohomology, Comm. in Algebra, 15 (1987), 53-121.

[6] W. Bosma and J. Cannon, Handbook of Magma Functions, Sydney: School of Mathematics and Statistics, University of Sydney, 1995.

[7] S. Bouc, The Dade group of a p-group, Invent. Math., 164 (2006), 189-231.

[8] J. Carlson, N. Mazza and D. Nakano, Endotrivial modules for finite groups of Lie type, J. Reine Angew. Math., 595 (2006) 93-120.

[9] J. Carlson and J. Thévenaz, Torsion endo-trivial modules, Algebras and Representation Theory, 3 (2000), 303-335.

[10] J. Carlson and J. Thévenaz, The classification of endo-trivial modules, Invent. Math. 158 (2004), no. 2, 389-411.

[11] E. Dade, Endo-permutation modules over p-groups I, Ann. Math. 107 (1978), 459-494.

[12] E. Dade, Endo-permutation modules over p-groups II, Ann. Math. 108 (1978), 317-346.

[13] S. Donkin, On Schur algebras and related algebras II, J. Algebra 111 (1987), 354-364.

[14] K. Erdmann, Young modules for symmetric groups, J. Austral. Math. Soc. 71 (2001), 201-210.

[15] D. Hemmer, J. Kujawa and D. Nakano, Representation type of Schur superalgebras, J. of Group Theory, 9 (2006), 283-306.

[16] D. Hemmer and D. Nakano, Support varieties for modules over symmetric groups, J. Algebra 254 (2002), 422-440. 
[17] A. Henke, On p-Kostka numbers and Young modules, European J. Combin. 26 (2005), no. 6, 923-942.

[18] G. James, The Representation Theory of the Symmetric Group, Lecture Notes in Mathematics No. 682, Springer, Berlin, 1978.

[19] A. Kerber, Representations of Permutation Groups I, Lecture Notes in Mathematics No. 240, Springer, Berlin, 1971.

[20] A. Kleshchev and J. Sheth, On extensions of simple modules over symmetric and algebraic groups, J. Algebra 221 (1999), 705-722.

[21] L. Weisner, On the Sylow Subgroups of the Symmetric and Alternating Groups, Amer. J. Math. 47 (1925), no. 2, 121-124.

Department of Mathematics, University of Georgia, Athens, Georgia 30602, USA E-mail address: jfc@math.uga.edu

Department of Mathematical Sciences, University of Aberdeen, Aberdeen, ScotLAND, UK

Department of Mathematics, University of Georgia, Athens, Georgia 30602, USA E-mail address: nakano@math.uga.edu 